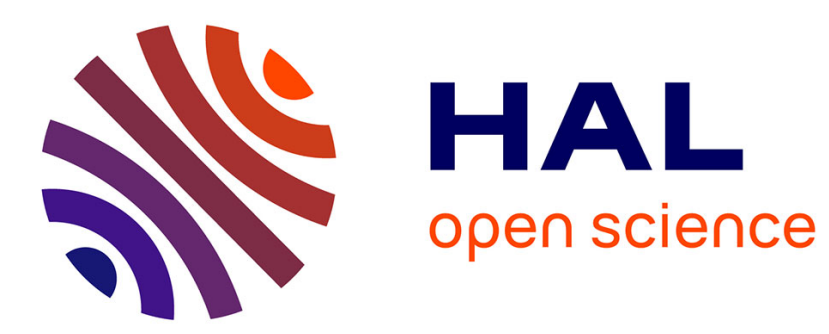

\title{
A Priori Relevance Based On Quality and Diversity Of Social Signals
}

Ismail Badache, Mohand Boughanem

\section{To cite this version:}

Ismail Badache, Mohand Boughanem. A Priori Relevance Based On Quality and Diversity Of Social Signals. ACM SIGIR Special Interest Group on Information Retrieval (SIGIR), Aug 2015, Santiago, Chile. pp.731-734. hal-01283948

\section{HAL Id: hal-01283948 \\ https://hal.science/hal-01283948}

Submitted on 7 Mar 2016

HAL is a multi-disciplinary open access archive for the deposit and dissemination of scientific research documents, whether they are published or not. The documents may come from teaching and research institutions in France or abroad, or from public or private research centers.
L'archive ouverte pluridisciplinaire HAL, est destinée au dépôt et à la diffusion de documents scientifiques de niveau recherche, publiés ou non, émanant des établissements d'enseignement et de recherche français ou étrangers, des laboratoires publics ou privés. 


\section{OATAO \\ Open Archive Toulouse Archive Ouverte}

\section{Open Archive TOULOUSE Archive Ouverte (OATAO)}

OATAO is an open access repository that collects the work of Toulouse researchers and makes it freely available over the web where possible.

This is an author-deposited version published in : http://oatao.univ-toulouse.fr/ Eprints ID : 14877

To link to this article : DOI : $10.1145 / 2766462.2767807$

URL : http://dx.doi.org/10.1145/2766462.2767807

To cite this version : Badache, Ismail and Boughanem, Mohand $A$

Priori Relevance Based On Quality and Diversity Of Social Signals. (2015) In: ACM SIGIR Special Interest Group on Information

Retrieval (SIGIR), 9 August 2015 - 13 August 2015 (Santiago, Chile).

Any correspondance concerning this service should be sent to the repository administrator: staff-oatao@,listes-diff.inp-toulouse.fr 


\section{A Priori Relevance Based On Quality and Diversity Of Social Signals}

\author{
Ismail Badache \\ IRIT Laboratory \\ University of Toulouse \\ Toulouse, France \\ Ismail.Badache@irit.fr
}

\author{
Mohand Boughanem \\ IRIT Laboratory \\ University of Toulouse \\ Toulouse, France \\ Mohand.Boughanem@irit.fr
}

\begin{abstract}
Social signals (users' actions) associated with web resources (documents) can be considered as an additional information that can play a role to estimate a priori importance of the resource. In this paper, we are particularly interested in: first, showing the impact of signals diversity associated to a resource on information retrieval performance; second, studying the influence of their social networks origin on their quality. We propose to model these social features as prior that we integrate into language model. We evaluated the effectiveness of our approach on IMDb dataset containing 167438 resources and their social signals collected from several social networks. Our experimental results are statistically significant and show the interest of integrating signals diversity in the retrieval process.
\end{abstract}

\section{Categories and Subject Descriptors}

H.3.3 [Information Storage and Retrieval]: Information Search and Retrieval

\section{General Terms}

Retrieval model, Experimentation

\section{Keywords}

Social signals; Signals diversity; Priors; Language models

\section{INTRODUCTION}

Majority of information retrieval systems (SIR) exploit two classes of sources of evidence to rank documents in response to the user query. The first class, the most exploited, is dependent on the query, it concerns the term statistics such as term frequency, distribution of term in documents. The second class concerns query-independent factors, which measures a kind of quality or importance of the document. Among these factors, the number of incoming links to a document [12], PageRank [5], topical locality [7], the presence of URL [17], document authors [13] and social signals [11].
In this paper we are interested in social signals associated to web resources. Nowadays, web pages include different social network buttons where users can express if they support or recommend content [1]. In this paper, we assume that, in addition to estimating a priori significance based on simply counting signals related to the resource [3], signals diversity associated to a resource can be also an index that indicates a resource interest beyond a social network or a community. The research questions addressed in this paper are the following:

1. How to estimate the signals diversity of a resource?

2. What is the impact of signals diversity on IR system?

3. Is there an influence of the social networks origin on the quality of their signals?

The remainder of this paper is organized as follows. Section 2 reviews some related work. Section 3 describes our social approach. In section 4 , we evaluate the effectiveness of our proposed approach and discuss the results. Finally, we conclude the paper and announce some future work.

\section{RELATED WORK}

While considerable work has been done in the context of social IR there is still a lack of user studies that would analyze the impact of users' actions diversity in a resource from diverse viewpoints. Major existing works [3, 6, 10, 11] focus on how to improve IR effectiveness by exploiting the users' actions and their underlying social network. For instance, Chelaru et al. [6] study the impact of social signals (like, dislike, comment) on the effectiveness of search on YouTube. In $[3,4]$, the authors show the impact of different time-sensitive signals taken into account individually and grouped as social properties without considering their diversity in the resource.

Our work is distinguished from the state of art. Our approach is based on signals diversity in the resource to improve relevance ranking of conventional text search. We exploit various signals extracted from different social networks. We note that in previous works, diversity has been applied only to the textual content of the document [2] [16].

Our goal is to estimate the significance of the resource by taking into account signals diversity into the resource. These sources of evidence are incorporated into language model that provides a theoretical founded way to take into account the notion of a priori probability of a document. 


\section{SOCIAL SIGNALS DIVERSITY}

Our approach consists in exploiting social signals and their diversity as a priori knowledge to take them into account in retrieval model. We rely on language model to model signals diversity as a prior probability.

\subsection{Notation}

Social information that we exploit within the framework of our model can be represented by 4 -tuple $<\mathrm{U}, \mathrm{R}, \mathrm{A}, \mathrm{SN}>$ where $U, R, A, S N$ are finite sets of instances: Users, Resources, Actions and Social Networks.

Resources. We consider a collection $C=\left\{D_{1}, D_{2}, \ldots D_{n}\right\}$ of $n$ documents. Each document (resource) $D$ can be a Web page, video or other type of Web resources. We assume that resource $D$ can be represented both by a set of textual keywords $D_{w}=\left\{D_{w_{1}}, D_{w_{2}}, \ldots D_{w_{z}}\right\}$ and a set of social actions $A$ performed on this resource, $D_{a}=\left\{D_{a_{1}}, D_{a_{2}}, \ldots D_{a_{m}}\right\}$.

Actions. We assume a set $A=\left\{a_{1}, a_{2}, \ldots a_{m}\right\}$ of $m$ actions that users can perform on the resources. These actions represent the relation between users $U=\left\{u_{1}, u_{2}, \ldots u_{h}\right\}$ and resources $C$. For instance, on Facebook, users can perform the following actions on resources: like, share, comment.

Social Properties. We consider a set $X=\{$ Popularity, Reputation $\}$ of 2 social properties that characterize a document $D$. Each property is quantified by a specific actions group. These properties are modeled as a priori probability.

\subsection{Query Likelihood and Document Priors}

We exploit language models [15] to estimate the relevance of document to a query. The language modelling approach computes the probability $P(D \mid Q)$ of a document $D$ being generated by query $Q$ as follows:

$$
P(D \mid Q) \stackrel{\text { rank }}{=} P(D) \cdot P(Q \mid D)=P(D) \cdot \prod_{w_{i} \in Q} P\left(w_{i} \mid D\right)
$$

$P(D)$ is a document prior, i.e. query-independent feature representing the probability of seeing the document. The document prior is useful for representing and incorporating other sources of evidence in the retrieval process. $w_{i}$ represents words of query $Q$. Estimating of $P\left(w_{i} \mid D\right)$ can be performed using different models (Jelineck Mercer, Dirichlet). The main contribution in this paper is how to estimate $P(D)$ by exploiting social signals.

\subsection{Estimating Priors}

According to our previous approach [3], the priors are estimated by a simply counting of actions performed on the resource. We assume that signals are independent, the general formula is the following:

$$
P_{x}(D)=\prod_{a_{i}^{x} \in A} P_{x}\left(a_{i}^{x}\right)
$$

$P_{x}\left(a_{i}\right)$ is estimated using maximum-likelihood:

$$
P_{x}\left(a_{i}^{x}\right)=\frac{\operatorname{Count}\left(a_{i}^{x}, D\right)}{\operatorname{Count}\left(a_{\bullet}^{x}, D\right)}
$$

To avoid Zero probability, we smooth $P_{x}\left(a_{i}\right)$ by collection $C$ using Dirichlet. The formula becomes as follows:

$$
P_{x}(D)=\prod_{a_{i}^{x} \in A}\left(\frac{\operatorname{Count}\left(a_{i}^{x}, D\right)+\mu \cdot P\left(a_{i}^{x} \mid C\right)}{\operatorname{Count}\left(a_{\bullet}^{x}, D\right)+\mu}\right)
$$

$P\left(a_{i}^{x} \mid C\right)$ is estimated using maximum-likelihood:

$$
P\left(a_{i}^{x} \mid C\right)=\frac{\operatorname{Count}\left(a_{i}^{x}, C\right)}{\operatorname{Count}\left(a_{\bullet}^{x}, C\right)}
$$

Where:

- $x \in\{P, R\}$ refers to the social property (popularity or reputation) estimated from a set of specific actions.

- $a_{\bullet}^{x}$ the total number of all social signals associated with $x$ property, in document $D$ or in collection $C$.

- $P_{x}(D)$ represents the a priori probability of $D$.

- $\operatorname{Count}\left(a_{i}^{x}, D\right)$ represents number of occurrence of specific action $a_{i}^{x}$ performed on a resource. $a_{i}^{x}$ designs action $a_{i}$ related (or used) to measure $x$ property.

We believe that the diversity of signals in a resource is an index that may indicate an interest beyond a social network or a community. Diversity and quantitative distribution of social signals in a resource may be considered as factors of relevance, i.e., a resource dominated by a single signal should be disadvantaged versus a resource with an equitable distribution of the signals. By applying diversity index of Shannon-Wiener [14], the corresponding formula is as follows:

$$
\operatorname{Diversity}_{s}(D)=-\sum_{i=1}^{m} P_{x}\left(a_{i}^{x}\right) \cdot \log \left(P_{x}\left(a_{i}^{x}\right)\right)
$$

Where $P\left(a_{i}^{x}\right)$ is defined above, and $m$ represents the total number of signals.

The Shannon index is often accompanied by Pielou evenness index [14] :

$$
\operatorname{Diversity}_{s}^{\text {Equit }}(D)=\frac{\text { Diversity }_{s}(D)}{M A X\left(\text { Diversity }_{s}(D)\right)}
$$

Where:

$$
M A X\left(\text { Diversity }_{s}(D)\right)=\log (m)
$$

The a priori probability $P_{x}(D)$ is estimated using the formula 4 multiplied by the diversity factor. The corresponding formula is as follows:

$$
P_{x}(D)=\left(\prod_{a_{i}^{x} \in A} P_{x}\left(a_{i}^{x}\right)\right) \cdot \text { Diversity }_{s}^{\text {Equit }}(D)
$$

\section{EXPERIMENTAL EVALUATION}

To validate our approach, we conducted a series of experiments on IMDb dataset. We evaluated the impact of signals diversity by combining it with language model.

\subsection{Description of Test Dataset}

We used a collection IMDb documents provided by INEX ${ }^{1}$. Each document describes a movie, and is represented by a set of metadata, and has been indexed according to keywords extracted from fields [3]. For each document, we collected

\footnotetext{
${ }^{1}$ https://inex.mmci.uni-saarland.de/tracks/dc/2011/
} 
specific social signals via their corresponding API of 5 social networks listed in table 2 . We chose 30 topics with their relevance judgments provided by INEX IMDb $2011^{2}$. In our study, we focused on the effectiveness of the top 1000 results. Table 1 shows an example of the documents containing social signals. The document URL is given by the following syntax: http: //www.imdb.com/title/ $\{i d\} /$

Table 1: Instances of 2 documents with social signals

\begin{tabular}{c|c|c|c|c}
\hline Id & Like & Share & Comment & +1 \\
\hline$t t 1730728$ & 30 & 11 & 2 & 0 \\
$t t 1922777$ & 12363 & 11481 & 20614 & 238 \\
\hline Id & \multicolumn{2}{|c|}{ Bookmark } & Tweet & Share(LIn) \\
\hline$t t 1730728$ & \multicolumn{2}{|c|}{0} & 2 & 0 \\
$t t 1922777$ & 12 & 2522 & 14 \\
\hline
\end{tabular}

Table 2 presents the social signals that we take into account in order to estimate each social property.

Table 2: Exploited social signals in quantification

\begin{tabular}{c|l|c}
\hline Property & Social signal & Network \\
\hline \multirow{5}{*}{ Popularity } & Number of Comment & Facebook \\
& Number of Tweet & Twitter \\
& Number of Share(LIn) & LinkedIn \\
& Number of Share & Facebook \\
\hline \multirow{3}{*}{ Reputation } & Number of Like & Facebook \\
& Number of Mention +1 & Google+ \\
& Number of Bookmark & Delicious \\
\hline
\end{tabular}

\subsection{Results and Discussions}

We conducted experiments with models based only on content of documents (Lucene Solr model ${ }^{3}$ and Hiemstra language model without prior [9]), as well as approaches combining content and social properties as a priori probabilities of document. We note that the best value of $\mu \in[90,100]$ The results are listed in table 3 .

Table 3 summarizes the results of precisions@ $k$ for $k \in$ $\{10,20\}$, nDCG (Normalized Discounted Cumulative Gain) and MAP (Mean Average Precision). In [3, 4], authors have already shown that taking into account the social characteristics without considering diversity improves IR compared to models based only on the topical relevance. In order to check the significance of the results, we performed the Student test [8] and attached * (strong significance against Baseline (A) and $(\mathrm{B})$ ) and $* *$ (very strong significance against Baseline (A) and (B)) to the performance number of each row in the table 3 when the $\mathrm{p}$-value $<0.05$ and $\mathrm{p}$-value $<0.01$ confidence level, respectively.

Before discussing the results we highlight that first we performed a correlation analysis by using the Spearman's correlation coefficient between signals diversity scores in the documents and their relevance. We found that the diversity represents a positive correlation with the relevance (Spearman's rho $=0.19)$, this result has aroused our interest and encourage us to exploit the diversity in our IR model. Table 3 (D) lists the results obtained by the integration of the signals diversity in the document. We notice that the NDCG and Precisions are generally better than the baseline listed in Table 3 (C) part when diversity is ignored.

We also notice that the both results are much more better than those obtained by topical models (Baseline (A) With-

${ }^{2}$ https://inex.mmci.uni-saarland.de/tracks/dc/2011/

${ }^{3}$ http://lucene.apache.org/solr/ out Priors) and Baseline (B) where signals are taken individually. This leads to our first conclusion, if multiple users of different social networks have found that a resource is useful, then it is more likely that other users will find these resources useful too.

Table 3: Results of $P @\{10,20\}, n D C G$ and MAP

\begin{tabular}{|c|c|c|c|c|}
\hline IR Models & $\mathrm{P} @ 10$ & $\mathrm{P} @ 20$ & nDCG & MAP \\
\hline \multicolumn{5}{|c|}{ (A) Baseline: Without Priors } \\
\hline Lucene Solr & 0.3411 & 0.3122 & 0.3919 & 0.1782 \\
\hline ML.Hiemstra & 0.3700 & 0.3403 & 0.4325 & 0.2402 \\
\hline \multicolumn{5}{|c|}{ (B) Baseline: Single Priors } \\
\hline Like & 0.3938 & 0.3620 & 0.5130 & 0.2832 \\
\hline Share & 0.4061 & 0.3649 & 0.5262 & 0.2905 \\
\hline Comment & 0.3857 & 0.3551 & 0.5121 & 0.2813 \\
\hline Tweet & 0.3879 & 0.3512 & 0.4769 & 0.2735 \\
\hline+1 & 0.3826 & 0.3468 & 0.5017 & 0.2704 \\
\hline Bookmark & 0.3730 & 0.3414 & 0.4621 & 0.2600 \\
\hline Share (LIn) & 0.3739 & 0.3432 & 0.4566 & 0.2515 \\
\hline \multicolumn{5}{|c|}{ (C) Baseline: Combination Priors } \\
\hline TotalFacebook & 0.4209 & 0.4102 & 0.5681 & 0.3125 \\
\hline Popularity & 0.4316 & 0.4264 & 0.5801 & 0.3221 \\
\hline Reputation & 0.4405 & 0.4272 & 0.5900 & 0.3260 \\
\hline All Criteria & 0.4408 & 0.4262 & 0.5974 & 0.3300 \\
\hline All Properties & 0.4629 & 0.4509 & 0.6203 & 0.3557 \\
\hline \multicolumn{5}{|c|}{ (D) With Considering Signals Diversity } \\
\hline TotalFacebook $^{\text {Div }}$ & $0.4227^{*}$ & $0.4187^{*}$ & $0.5713^{*}$ & $0.3167^{*}$ \\
\hline Popularity ${ }^{D i v}$ & $0.4403^{* *}$ & $0.4288^{* *}$ & $0.5983^{* *}$ & $0.3320^{* *}$ \\
\hline Reputation $^{\text {Div }}$ & $0.4480^{* *}$ & $0.4306^{* *}$ & $0.6110^{* *}$ & $0.3319^{* *}$ \\
\hline All Criteria ${ }^{D i v}$ & $0.4463^{* *}$ & $0.4318^{* *}$ & $0.6174^{* *}$ & $0.3325^{* *}$ \\
\hline All Properties ${ }^{D i v}$ & $0.4689^{* *}$ & $0.4563^{* *}$ & $0.6245^{* *}$ & $0.3571^{* *}$ \\
\hline
\end{tabular}

\subsection{Quantitative and Qualitative Analysis}

To better understand the effect of these social signals on the selection of relevant documents, we analyze their distribution in the all documents returned by 30 topics.

According to Table 4 we notice that the average frequency of the signals in the relevant documents is higher compared to irrelevant documents (ex. the average of like is 362 actions in the relevant documents while in irrelevant documents is 61 actions). We also note that the signals coming from Facebook capture the highest number of relevant documents (see Figure 2), knowing that they are many in irrelevant documents but with a much smaller average. This is due to the engagement rate on Facebook and its dynamic growth [1]. Therefore, the distinction between relevant documents and irrelevant documents is much more sensitive to signal frequency, i.e., relevant documents are characterized by a very high number of Facebook signals compared to the irrelevant documents (see Figure 1). The signals tweet and +1 come in second position with an average frequency, respectively, 97 and 29 actions in the relevant documents (see Figure 1). The signal of Delicious (Bookmark) is the weakest criterion among these signals, it appears in 429 relevant documents with an average frequency of 13 actions per document only. Concerning the signal of LinkedIn, we note that $95 \%$ of its actions of share are condensed in 601 relevant documents with an average frequency of 67 actions per document. However, the number of relevant documents captured by Linkedin share signal is very low compared to that captured by Facebook signals, this is due to LinkedIn engagement rate which is very low compared to Facebook, but LinkedIn share signal represents the most reliable source in terms of trust compared to other social signals. Therefore, we can say that the presence of this signal, whatever its frequency in a document represents a relevance index. 
Table 4: Statistics on the distribution of the signals in the documents (relevant and irrelevant)

\begin{tabular}{|c|c|c|c|c|c|c|}
\hline & \multicolumn{3}{|c|}{ Relevant documents containing signals } & \multirow{2}{*}{$\frac{\text { Relevant documents without signals }}{\text { Number of documents }}$} & \multicolumn{2}{|c|}{ Irrelevant documents } \\
\hline & $\begin{array}{l}\text { Number of } \\
\text { documents }\end{array}$ & $\begin{array}{c}\text { Number of } \\
\text { actions }\end{array}$ & Average & & $\begin{array}{c}\text { Number of } \\
\text { actions }\end{array}$ & Average \\
\hline Like & 2210 & 800458 & 362.1981 & 555 & 1678040 & 61.6133 \\
\hline Share & 2357 & 856009 & 363.1774 & 408 & 1862909 & 68.4012 \\
\hline Comment & 1988 & 944023 & 474.8607 & 777 & 1901146 & 69.8052 \\
\hline Tweet & 1735 & 168448 & 97.0884 & 1030 & 330784 & 12.1455 \\
\hline+1 & 790 & 23665 & 29.9556 & 1975 & 49727 & 1.8258 \\
\hline Bookmark & 429 & 5654 & 13.1794 & 2336 & 20489 & 0.7523 \\
\hline Share (LIn) & 601 & 40446 & 67.2985 & 2164 & 2341 & 0.0859 \\
\hline \multicolumn{5}{|c|}{ Total relevant documents : 2765} & \multicolumn{2}{|c|}{ Total irrelevant documents : 27235} \\
\hline
\end{tabular}

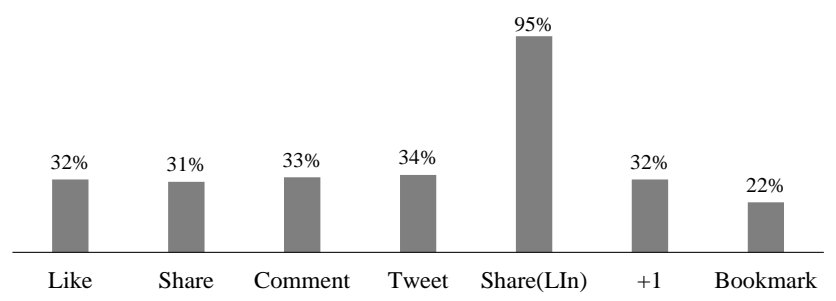

Figure 1: Signals \% in the relevant documents

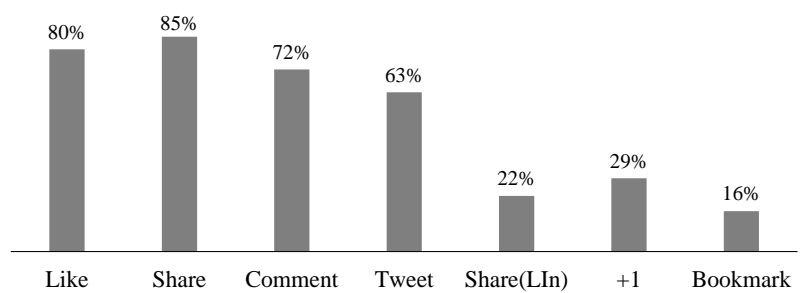

Figure 2: Relevant documents \% containing signals

Finally, according to this preliminary statistical study, we observe that each social network has its own specific influence on the quality of its social signals. The quality of signals, provided by Facebook, Twitter, Google+ and Delicious, in a document depend on their frequencies, the more the signals are frequent on the resource, the more its a priori importance increases. However, the LinkedIn signal does not only depend on its frequency in the document because it has in itself a power of mature trust compared to the other signals. This amounts to the maturity of LinkedIn users who are well reputed compared to other social networks users.

\section{CONCLUSION}

We proposed in this paper a search model based on users' actions associated with a document. We proposed to estimate a social priors of a document by considering signals diversity. The proposed model is based on language model that incorporates this a priori knowledge. Experimental evaluation conducted on IMDb dataset shows that taking into account these social features in a textual model improves the quality of returned search results. Finally, to investigate the influence of social networks on the quality of signals, we developed a statistical study on the distribution of social signals for each social network in both relevant and irrelevant documents.

For future work, we plan to address some limitations of the current study. We plan to integrate other social data into proposed approach. Further experiments on another dataset are also needed. This is even with these simple elements, the first results encourage us to invest more this track.

\section{REFERENCES}

[1] O. Alonso and V. Kandylas. A study on placement of social buttons in web pages. arXiv, 2014.

[2] A. Angel and N. Koudas. Efficient diversity-aware search. In SIGMOD, pages 781-792, 2011.

[3] I. Badache and M. Boughanem. Social priors to estimate relevance of a resource. In IIiX, 2014.

[4] I. Badache and M. Boughanem. Document priors based on time-sensitive social signals. In ECIR, pages 617-622, 2015.

[5] S. Brin and L. Page. The anatomy of a large-scale hypertextual web search engine. In $W W W$, pages 107-117, 1998.

[6] S. Chelaru, C. Orellana-Rodriguez, and I. S. Altingovde. How useful is social feedback for learning to rank youtube videos? $W W W$, pages 1-29, 2013.

[7] Brian D Davison. Topical locality in the web. In SIGIR, pages 272-279, 2000.

[8] William Sealy Gosset. The probable error of a mean. Biometrika, 6(1):1-25, 1908. Originally published under the pseudonym "Student".

[9] D. Hiemstra. A linguistically motivated probabilistic model of information retrieval. In $E C D L$, pages 569-584, 1998.

[10] G. Kazai and N. Milic-Frayling. Effects of social approval votes on search performance. In ITNG, pages 1554-1559, 2009.

[11] A. Khodaei and C. Shahabi. Social-textual search and ranking. In CrowdSearch, pages 3-8, 2012.

[12] W. Kraaij, T. Westerveld, and D. Hiemstra. The importance of prior probabilities for entry page search. In SIGIR, pages 27-34, 2002.

[13] C. Macdonald and I. Ounis. Voting for candidates: adapting data fusion techniques for an expert search task. In CIKM, pages 387-396, 2006.

[14] EC. Pielou. Shannon's formula as a measure of specific diversity: its use and misuse. American Naturalist, pages 463-465, 1966.

[15] Jay M. Ponte and W. Bruce Croft. A language modeling approach to information retrieval. In SIGIR, pages 275-281, 1998.

[16] L. Qin, J. X Yu, and L. Chang. Diversifying top-k results. VLDB Endowment, 5(11):1124-1135, 2012.

[17] T. Westerveld, W. Kraaij, and D. Hiemstra. Retrieving web pages using content, links, urls and anchors. 2002. 